\title{
Erwiderung auf die „Bemerkung"t des Hrn. E. Ludwig zu meiner Arbeit „Ueber die Ausscheidung des Queck- silbers" "), nebst weiteren Aufschlüssen über diese Ausscheidung,
}

\author{
Von Dr. Schuster, pr. Arzt in Aachen.
}

Herr Prof. E. Ludwig orgeht sich in genannter "Bemerkung" wegen meiner angeblichen "Anklage" gegen seine Methode des Qnecksilbernachweises in thierischen Substanzen. Von einer solchen "Anklage" der Unzuverlässigkeit kann meinerseits um so weniger die Rede sein, als ich in meiner Arbeit ja angegeben habe, dass die E. Ludwig'sche Methode, wenn auch in der von FürbringerSchridde modificirten Weise, in den ron mir veranlassten Untersuchungen zur Anwendung gekommen ist. Gegen diese Modification ist bisher von keiner Seite Einspruch erhoben, und daher wie es scheint auch von Herrn E. Ludwig deren Werth zugegeben; ibr liegt aber das Ludwig'sche Verfahren zu Grunde. Ich bin demnach der Letzte, welcher die grossen Verdienste des Herrn E. Ludwig um den Nachweis des Quecksilbers in thierischen Substanzen nicht anerkennen sollte.

Aus den hiesigen Analysen über die Ausscheidung des Quecksilbers, die noch fortgesetzt werden, kam ich bis jetzt zu dem Resultate, dass sich 8 Monate nach Beendigung der selbst 50malige Anwondung der grauen Salbe umfassenden Kur das Quecksilber weder im Harn, noch in den Fäces nachw cisen liess. Das Qnecksilber wurde verhältnissmässig seltener im Harn bei meinen Thllon nachgewiesen, als in den im Ludwig'schen Laboratorium unter-

1) S. diese Vierteljahrschr. 1882, 1. Heft. 
suchten. Das musste mir anffallen einerseits, weil ich es in den bis jetzt untersuchten Fäces jetzt regelmässig fand von solchen Patienten, bei denen im Harne gegen Erwarten Mercur nicht gefunden wurde, weil andererseits im Ludwig'schen Laboratorium die Herren Vajda und Paschkis 2, 3, 5, 7, 13 Jahre nach verhältnissmässig wenigen (17maligen) Mercuranwendungen Quecksilber im Harne gefunden hatten.

Was lag da wohl näher als nach dem Grunde dieses grossen Unterschiedes in den Untersuchungsresultaten zu suchen?

Dass die genannten Herren das Ludwig'sche Verfahren genau durchgeführt hatten, daran konnte kein Zweifel aufkommen, ich habe dies in meiner Arbeit bereits derartig betont, dass Herr E. Ludwig sich der nochmaligen Hervorhebung über die grosse Sicherheit, mit der die Untersuchmgen in seinem Laboratorinm umgeben sind, hätte entschlagen dürfen. Entweder musste ich also den Gegensatz meiner und der genannten Resultate einfach bestehen lassen, oder nach einem Aufschlusse suchen. Dass ich letzteres gethan habe, wird mir Niemand, anch Herr E. Ludwig nicht übel nehmen. Aber dass ich es wagte, in dem von ihm zu seinem Verfahren neben dem Kupfer auch empfohlenen Zinkstaube einen Anhalt als Einwurf gegen die genannten Resultate zu suchen, das brachte mir die E. Ludwig"sche „Bemerkung" oin.

Ich behauptete „Jeder im Handel vorkommende Zinkstaub enthält Arsenik".

Aachen befindet sich umgeben von Zinkhütten, in denen Zinkstaub in grösster Menge gewonnen wird; hiedurch war die Untersuchung des Zinkstaubes verschiedensten Ursprunges erleichtert. Demnach hatte jene Behauptung meinerseits nichts Auffälliges; zudem ist sie wahr. Das wird Herr Prof. Ludwig auch nicht bestreiten.

Ich behauptete zweitens "dass Jod mit Arsen eine dem Jodquocksilber in ihrer rothen Farbe sehr ähnliche Verbindung geben kann".

Dagegen erwähnt Herr L. Ludwig, es sei jedem Anfänger in der analytischen Chemie bekannt, dass die Jodide genannter Metalle ihrem äussoren Ansehen nach einen "gewissen Grad" von Aehnlichkeit haben können. Ich aber sage aus dem Nachweise des schön rothen Jodarsens, dass die Jodide beider Metalle, besonders wenn es sich um sehr kleine Mengen handelt, geradezu 
sich makroskopiseh nicht unterscheiden lassen. Das ist also bei Weitem mehr, als "ein gewisser Grad" von Aehnlichkeit. Aber 'nun kommt erst der Kernpunkt der Ludwig'schen Abfertigung.

Ich meinte nun zur Erklärung des Gegensatzes der erwähnten Resultate, der Einwand sei zulässig, dass also unter Benutzung des Zinkstaubes bei dem Ludwig'schen Verfahren auch der Arsenik einen rothen Jodbeschlag geben könnte.

Darauf erwiedert nun Herr E. Ludwig - und zwar mit weit grösserer Berechtigung - dass ich mit Zinkstaub und normalem Harn oder Wasser genau nach seinem Verfahren (unter Anwendung des Luftstromes) einmal Versuche machen solle. Ich fühlte mich bereits im Februar d. J. veranlasst, mehrere solcher Versuche machen zu lassen; sie schlugen negativ aus. Natürlich! Im Luftstrome oxydirt sich das im Zinkstaube enthaltene Arsenik und gibt in Folge dessen mit Jod kein Jodarsen.

Ist aber darum mein Einwand hinfällig? Durchaus nicht! Denn es kann dennoch metallisches Arsen sich unter zwei Bedingungen verfüchtigen, und zwar 1. durch Stockung des Luftstromes, so dass nicht alles Arsen verbremen kann; 2. durch die reducirende Wirkung der etwa im Harne vorhandenen organischen Substanzen. Man hat nämlich nicht immer mit "normalem" Harn bei dessen Untersuchung auf Quecksilber zu thun. Das sind Verhältnisse, die nach Rücksprache nicht mit „irgend Jemand“ sondern mit ihr Fach beherrschenden Chemikern nach deren Ansicht als möglich angesehen werden müssen und für deren Giltigkeit einzustehen und sie zu vertheidigen, einer derselben, der bereits wiederholt erwähnte Fach-Chemiker, Horr Dr. Schridde, sich hiermit bereit erklärt. Ich muss daher meine über den Werth des Zinkstaubes der Lametta gegenüber ausgesprochene Ansicht aus den angegebenon Gründen anfrecht erhalten. Bemorke noch ferner, dass in Stolberg bei Aachen selbst Galmei verhüttet wurde, der stark zinnoberhaltig war.

Nun kommt dagegen allerdings die Erklärung E. Ludwig's, dass die von mir als möglich gehaltenen Fehlerquellen in seinem Laboratorium selbstrerständlich ausgeschlossen werden, dass die in seinem Laboratorium erzielten Resultate über Ausseheidung des Quecksilbers nicht angezweifelt werden dürfen. 
Nun! dann trifft hiernach für diese Fälle meine Erklärung des Gegensatzes in meinen und den Vajda-Paschki s'schen Resultaten nicht zu, es bleibt dann zunächst dieser Gegensatz also unaufgeklärt bestehen, und weitere Untersuchungen müssen dann hierüber Licht suchen.

Bis heute sind auf meine Veranlassung einige zwanzig $\mathrm{Fä-}$ caluntersuchungen neben vielen Harnuntersuchungen auf Quecksilber vorgenommen worden bei Patienten, welehe sich entweder noch in der Quecksilberbehandlung befanden, oder welche vor kürzerer oder längerer Zeit bis zu einem Jahre vorher unter meiner Leitung zu ihrer Kur $40 \mathrm{Mal}$ und mehr die graue Salbe angewendet hatten.

Ausser den in meiner Arbeit über die Ausscheidung des Quecksilbers bereits erwähnten 7 Fäcaluntersuchungen sind bis heute noch vierzehn weitere gemacht worden. Dieselben dürften für die Frage von der Ausscheidung des Quecksilbers von besonderem Interesse sein; deshalb erlaube ich mir, sie hier anzuführen.

Bei Fall 36 (s. m. Arb. I. Heft, 1882) wurden noch dreimal die Fäces theils während, theils 6 und 10 Tage nach der Kur untersucht. Dieselben waren jedesmal stark Hg-haltig; das vorletzte Mal zeigte sich das $\mathrm{Hg}$ in quantitativ leicht zu bestimmender Menge. Deshalb wurde einige Tage später eine dritte Untersuchung vorgenommen zu dem Zwecke, um zu erfahren, ob das $\mathrm{Hg}$ in den Fäces in gelöstem oder ungelöstem Zustande vorkomme. Der wässerige Auszug ergab ein negatives Resultat; der feste Rückstand dagegen wieder ein schönes positives Resultat. In diesem Falle war also das Hg ungelöst in den Fäces erhalten. Es konnte demnach entweder als Calomel, Schwefelquecksilber oder metallisches Quecksilber vorhanden sein, insofern man nicht eine unlösliche, complicirte, organische Verbindung annehmen will.

Fall 19, der einmal einen $\mathrm{Hg}$-haltigen, einmal einen $\mathrm{Hg}$ freien Harn darbot, der sich nach zweimonatlicher Abwesenheit mit Gesicht- und Pharynx-Ulcera wieder vorstellte, zeigte bei den vom 22. März bis jetzt 5 Mal in Zwischenräumen von 6 bis 14 Tagen vorgenommenen Fäcalunterstichungen - die letzte wurde 4 Monate nach der Kur vorgenommen, ohne dass in dieser Zeit Mercur gebrancht worden wäre - jedesmal $\mathrm{Hg}$ und zwar in jedesmal sich 
vermehrender Menge. Dagegen fohlte Letzteres bei einer gleichzeitig vorgenommenen Harnuntersuchung. Der Kranke gebraucht während seines Hierseins den Thermalbrunnen und Bäder.

In drei Fällen wurden noch die Fäces acht Monate nach der Behandlung whtersucht. Letztere hatte in 45 resp. 50 Einreibungen von je 5 Gramm grauer Salbe bestanden. Das Resultat war jedesmal ein negatives. In zwei von diesen Fällen wurde anch der Harn gleichzeitig auf Quecksilber untersucht; das Resultat war ein negatives.

Dagegen darf folgender vor 10 Tagen zuerst untersuchter Fall nicht verschwiegen werden: Herr $R$. hat in einem Zeitraume von drei Monaten wegen eines specifischen Cerebrospinalleidens 46 Inunctionen grauer Salbe gebraucht. Die jetzt untersuchten Fäces, sowie der Harn zeigten sich Hg-frei. Die untersuchten Fäces waren spärlich. Immerhin war er die erste Ausnahme unter den allein bis jetzt positiv ausgefallenen Untersuchungen der Fäces. Es wurde deshalb nach 8 Tagen, während welcher Zeit noch 4 Innunctionen gemacht wurden, eine nochmalige Untersuchung der Fäces vorgenommen. Da an einem Tage sebr wenig Fäcalmasse entleert wurde, so wurde die von drei Tagen erhaltene nicht zu grosse Menge untersucht. Diesmal zeigt sich reichlich $\mathrm{Hg}$ in den Fäces.

Die weiteren bis jetzt untersuchten Fälle bestätigen also vollauf die in meiner ersten Arbeit über die Ausscheidung des Quecksilbers ausgesprochenen Schlusssätze. Das Quecksilber wird durch dio Fäces in beständiger, regelmässiger Weise ausgeschieden. Vier Monate nach der Kur wurden die Fäces noch Hg-haltig gefunden. Acht Monate nach längerer Hg-kur konnte bis jetzt weder im Harn noch in den Fäces $\mathrm{Hg}$ nachgewiesen werden; mit Rücksicht darauf, dass viel weniger $\mathrm{Hg}$ täglich ausgeschieden, als während der Kur täglich aufgenommen wird, ist die Möglichkeit zuzugeben, dass auch einmal acht Monate nach lange fortgesetzter Kur boi regelmässig vor sich gehender Ausscheidung durch die Fäces das Quecksilber in denselben nachgewiesen werde.

Aachen, den 5. Mai 1882. 